\title{
Diálogo convivial con El lugar de Saer ${ }^{1}$
}

\author{
Ricardo Piglia \\ Universidad de Buenos Aires
}

El 14 de agosto de 2007 se inauguraba el III Argentino de Literatura organizado por la Dirección de Cultura y el Departamento de Letras de la Facultad de Humanidades y Ciencias (FHUC) de la Universidad Nacional del Litoral (UNL). Por tercer año consecutivo la literatura salía de los claustros para generar un espacio de discusión y reflexión en torno a las prácticas literarias y sus múltiples vertientes, y para escuchar las voces de sus más importantes representantes. En esa oportunidad el invitado de honor fue Ricardo Piglia.

El Centro de Estudios Comparados de la Facultad de Humanidades y Ciencias fue convocado hace unos ańos para desgrabar y publicar los registros de ese evento. Distintas circunstancias postergaron hasta hoy esa tarea. Si bien el libro con los resultados de ese encuentro está en edición ${ }^{2}$, proponemos, a modo de Convivio, incluir algunos fragmentos de la conferencia de Piglia por la perspectiva comparada y local que propone su lectura sobre Saer.

Como todo acto efímero, una conferencia — a pesar de haber sido grabada - es un conjunto de signos y de vivencias que solo puede atesorar quien estuvo presente en ese allí y ese ahora, que ya es irremediable pasado. El texto que desgrabamos y que en parte reproducimos en este número celebratorio de nuestra revista tiene la intención de continuar con el deseo que el mismo Piglia expresó antes de empezar el debate con el público: mantener un diálogo, un Convivio, con un ahi, que todavía permanece y que puede ser en parte recuperable, en la sostenida levedad de la voz y la lectura.

Este diálogo comenzó en 1960 (hace exactamente 60 años), cuando Juan José Saer publicaba su primer libro: En la zona. Pensar la zona desde Saer es concebir una tradición de un ahora y un aqui que nos justifica. Aportar a las discusiones que tuvieron como protagonista a Saer, sus personajes, Piglia y a la Universidad Nacional del Litoral (con Santa Fe como entorno) como espectadores privilegiados, es entonces nuestro deber.

El mismo Piglia enuncia y justifica este modo localmente dialógico al informarnos cómo desarrolló su conferencia: improvisando y no leyendo. Por lo que, si luego el texto «El lugar de Saer» fue publicado por su autor con otros protocolos y en otros contextos, el contenido de esta conferencia cumple con la fascinación y la novedad de la performance y de la unicidad de la oralidad.

Así lo cristaliza el conferencista al momento de terminar su discusión y esperar las preguntas del público: 
Piglia: Hay que devolver las preguntas... Eso fue por no leer la conferencia. Preferí no leer la conferencia. Me pareció que eso nos iba a permitir una... un riesgo mayor de parte mía y también porque me parece que ese es un poco el recuerdo que tengo de la conversación que tuvimos con Saer, que se publicó aquí en una colección de la Universidad, que creo fue en el año ' $86 /$ ' $87^{3}$. Y que hicimos como los payadores, ¿¿no? y me parece que siempre hay que, en cierto sentido, hay que recuperar esa cuestión de estar ahí. Como decía Macedonio Fernández, ¿no? Macedonio siempre decía «no me vine preparado para improvisar». Entonces, yo me vine preparado para improvisar, pero traté de, bueno, nada, de establecer con ustedes un modo de acercarme a la obra de Saer, que admiro muchísimo, planteando estas cuestiones que son apenas algunas aproximaciones, ¿̨no es cierto? Desde luego que podemos conversar acerca de lo que yo he dicho, y de otra cosa. No necesariamente las preguntas tienen que ser, tienen que estar ligadas, a lo que hablamos aquí, ahora.

Reproducimos solo una de las preguntas del debate porque se engarza con una parte de la conferencia que incluimos en este Convivio. Y que responde al modo como Piglia pensaba la construcción de la genealogía que cada escritor «se hace» para inventarse su propia tradición:

Moderador: La siguiente pregunta hace referencia al modo en que se construyen esas tradiciones, o esas genealogías de las que hablabas hoy, al hacer referencia a la literatura de Juan José Saer. Piglia: Bueno, desde luego que me parece que cuando uno, por ejemplo... es muy común que cuando uno nombra un escritor que le gusta mucho, no quiere decir que ese escritor forme parte de su propia tradición, ¿̨no? Muchas veces, uno nombra escritores, pero el problema es qué tipo de escritores son los que realmente permiten narrar, le permiten a uno, digo, tener la sensación de que es posible seguir narrando, ¿̨no? Yo siempre uso una frase de Shklovski, un escritor y crítico ruso que decía «las musas son la tradición literaria». Es decir que hay una tradición previa, y para mí el ejemplo más claro de esto es la literatura norteamericana. La literatura norteamericana es una literatura que trabaja la tradición de una manera espontánea muy clara. Hemingway dice "yo vengo de Mark Twain, no vengo de la prosa alta a la inglesa, de la prosa bíblica de Faulkner, yo vengo del habla» dice Hemingway, ¿̨no? Entonces, dice «la literatura norteamericana empieza con Mark Twain», como diciendo «es Mark Twain». Y después, si le preguntan qué escritor le gusta, él va a decir que le gusta Melville y que le gustan otros escritores, pero que no tienen el mismo tipo de uso. Entonces, habría que hacer una distinción entre los escritores que uno admira y los escritores con los cuales uno tiene relaciones más productivas, ¿̨no? Uno puede admirar mucho un escritor, pero ese escritor no funciona en relación a lo que uno está... Por ejemplo, para mí ha sido muy productiva la relación con el género policial. Es decir, el modo, la forma de relato como investigación me interesa mucho, y el género policial, desde luego, estructura eso. Se empieza con algo que no se entiende. Y se empieza a reconstruir esa historia perdida, para ver qué pasa. Puede ser un crimen o no. Entonces, ahora si me preguntan qué escritores son los que admiro, bueno, voy a decir que me gusta Chandler. Pero, desde luego, hay escritores a los que admiro y considero más importantes. Pero para mí es más importante quizás ese tipo de cuestión. Entonces, esa es una manera de entender la cuestión. La otra es que las tradiciones tienen que ver con guerras y peleas y luchas de poéticas. La literatura no es un campo neutral, como se suele pensar, ¿̨no? Es un campo de enfrentamientos y de tensiones. $Y$ en ese juego de poéticas y de tensiones nosotros pensamos Puig, Saer, Walsh. Ahí podemos ver, claramente, las tensiones que ustedes pueden imaginar. Si nosotros leemos a Saer desde Puig, no queda nada de Saer. Y si leemos a Puig desde Saer, tampoco queda nada. Es decir, las poéticas anulan los otros textos. Es decir, 
producen un efecto que es un efecto, digamos, cómo decirlo, de descarte. Entonces eso es muy interno, me parece a mí, al modo en que los escritores leemos. Leemos a los otros o leemos a los escritores a partir de los cuales construimos la genealogía. Entonces, supongamos Puig ¿qué genealogía construía? Los films de Hollywood de los años '30. Y decía que, por ejemplo... fíjense con Joyce, dijo una cosa Puig que me pareció extraordinaria. Dijo «yo miré el Ulises y vi que todos los capítulos estaban escritos con una técnica distinta, y eso ya me alcanzó». Y así lee un escritor. Puig se dio cuenta que era posible escribir una novela como la que él escribía, donde en cada capítulo hay una técnica nueva, distinta. Es decir, los usos de la literatura no tienen que ver con una lectura donde uno lee todo y completamente, sino que son usos, son movimientos muy particulares, que tienen que ver con la tradición y la genealogía, ¿no?

Invitamos entonces a la lectura de un fragmento de la conferencia desarrollada por Piglia en ese III Convivio Argentino con la literatura, en alocución transcripta que conserva los ritmos y matices de oralidad dados por el orador:

\section{Fragmentos de la conferencia de Ricardo Piglia: El lugar de Saer}

Ricardo Piglia: Bueno, les agradezco a todos que hayan venido esta noche y me siento muy honrado de estar aquí, y también desde luego es una responsabilidad para mí hablar de Saer en Santa Fe. Desde luego, el título de la conversación - yo diría, mejor, más que de la conferencia-, el título «El lugar de Saer» tiene distintas lecturas posibles. La primera lectura tiene que ser literal, el lugar de Saer es este, es decir, el lugar de Saer es Santa Fe. Con esto quiero decir que debemos rescatar, como un punto de partida de la obra de Saer, la fidelidad a un lugar. La fidelidad a un espacio. Que por un lado lo conecta con algunos escritores que están siempre en diálogo con su obra, como es el caso de Pavese, y esta relación de Pavese con el Piamonte me parece que ha sido muy fundamental para la construcción de ciertos textos de Saer. Esas novelas cortas de Pavese que le gustaban muchísimo a Saer, donde un grupo de amigos, en una ciudad de provincia, pasan la noche conversando literatura. Eso que en Pavese insiste en sus textos es algo que Saer supo leer muy bien, del mismo modo, desde luego, que supo leer muy bien a Faulkner, la idea de la invención de un lugar. La construcción de la ficción es antes que nada una espacialización, la definición de un territorio es el punto de partida de la ficción. Yo diría que Saer es quizá el escritor al que mejor le funciona la recomendación de Nabokov, ¿no?, que decía [que] siempre que leemos tenemos que hacer un mapa. "Tenemos que leer siempre haciendo un mapa", decía Nabokov, cuando trataba de definir a los buenos lectores de la ficción, es decir, tenemos que tener claro el espacio donde la ficción sucede y debemos tratar, decía Nabokov, debemos 
tratar de visualizar el espacio dentro del cual la narración sucede. Me parece que Saer es, en ese sentido, un gran maestro. Y todos debemos hacer a veces algunos mapas.

Hoy mismo estábamos en el Hotel Castelar, creo que es - espero que esto no sea publicidad-, estábamos en el Hotel Castelar y en ese hotel empieza una de las primeras novelas de Saer: La vuelta completa. Y enfrente está el correo y enfrente estaba la vieja estación de ómnibus y por lo tanto esa espacialización que va a funcionar en sus textos como un elemento básico es un modo de definir un territorio. Me parece entonces que cuando decimos el lugar de Saer, estamos pensando antes que nada en un escritor que ha sido muy fiel a una zona, y debemos valorar esa decisión. Sobre todo debemos valorar esa decisión en este momento, donde [cuando] a menudo muchos escritores contemporáneos creen que deben escribir literatura mundial, es decir, que deben empezar pensando en temas que pueden interesas a públicos muy diversos, y entonces empiezan a localizar sus novelas en aeropuertos, en ciudades prestigiosas, con temas que se supone que son conocidos por todos. Mientras que Saer sería un ejemplo de lo que la verdadera literatura hace, que es fijarse en un punto, trabajar a partir de un espacio muy definido y construir a partir de ese espacio historias que pueden llegar luego a cualquier ámbito. De modo que en un momento en el que la literatura parece correr el riesgo de estar atrapada por la idea de esta especie de mercado mundial de circulación de bienes y de productos simbólicos, me parece que el ejemplo de Saer, como el de otros, desde luego, debe servirnos como punto de partida para abrir una discusión. En el sentido en que Saer ha sido siempre alguien que ha planteado la necesidad de trabajar a partir de un espacio conocido, un espacio que tenga siempre una manera de funcionar, que sintamos que el que lo narra lo tiene como un espacio muy próximo. Entonces ese sería el primer sentido del título «El lugar de Saer», esta idea de que un escritor está siempre situado y que sus textos se sitúan en un espacio determinado.

Desde luego la noción misma de lugar, no hace falta decirlo, forma parte para Saer de una suerte de poética, no se trataría solamente de una cuestión de tema, no se trata solamente de una decisión temática en Saer: «voy a situar mis historias en un territorio definido y voy a escribir la historia de ese territorio». Sino que también se trata de una definición que hace a una poética de la narración, por eso el último libro que Saer publica en vida se llama Lugar, y el primer libro que Saer publica, como sabemos, se llama En la zona. De modo que también ahí podemos ver el lugar ya no solamente como un tema sino como una posición respecto a qué quiere decir narrar. Narrar quiere decir fundar un espacio. Y en este movimiento de fundar un espacio me parece que Saer realiza una operación muy original porque el espacio que construye, que es el espacio de la ciudad de Santa Fe, como sabemos, y la zona del litoral que rodea la ciudad de Santa Fe, es muy identificable como tal, pero Saer nunca nombra a la ciudad como la ciudad de Santa Fe. Podríamos decir que de esa manera construye una suerte de mundo, que es el mundo real pero también es un mundo paralelo. No llama a ese espacio con un nombre ficcional, no realiza la operación de Faulkner de llamar Yoknapatawpha, ¿no? al espacio de la ficción o Santa María, como es el caso de Onetti. Sitúa perfectamente en calles identificables pero nunca llama a la ciudad en la que suceden las historias, nunca la llama Santa Fe. Es decir, le da siempre esa especie de espacio para que la ficción pueda encontrar su lugar propio. De modo que esta idea del lugar y el espacio, como 
formando parte de su poética narrativa, me parece que tendría que ser también un elemento para pensar sobre la originalidad de la perspectiva narrativa de Saer.

Y el tercer sentido que tiene, para mí, esta idea del lugar de Saer me parece que surgió recién en la cita que nos recordaba su interés en la tradición. Porque para mí también la pregunta sobre el lugar de Saer es el lugar de Saer en la tradición, qué lugar tiene Saer en la tradición y cuál es la tradición que Saer construye. Y como él mismo decía en la cita que escuchábamos recién, un escritor construye una red, construye una determinada genealogía y por lo tanto siempre narramos en el interior de una tradición. Lo sepamos o no, siempre estamos narrando a partir de una tradición, es decir, alguien ha narrado antes y por lo tanto, narramos en el espacio que otros antes han construido para que narrar sea posible. Es imposible narrar sin tradición. Y por lo tanto, si uno no tiene una tradición tiene que inventarla. Tiene que encontrar el modo de construir esa suerte de genealogía imaginaria y me parece que la obra de Saer nos permite pensar esta cuestión: ¿cuál es la tradición al interior de la cual Saer se instala y qué tipo de tradición es la que él define? Me parece que es un punto central. Yo recuerdo que cuando lo conocí... yo lo conocí en 1964 en una mesa redonda, en la Facultad de Filosofía y Letras de la calle Viamonte, en la legendaria Facultad de Filosofía de la calle Viamonte. Como sabemos, todas las facultades de Filosofía son legendarias, pero aquella tiene el prestigio de aquella zona, que era una zona fronteriza con los piringundines y los bares del bajo de Buenos Aires. De modo que la facultad estaba al mismo tiempo en el borde, donde estaban las alternadoras y donde estaban las boites de la zona del puerto. Entonces fíjense cómo eran las cosas en la literatura argentina en aquellos tiempos que yo estaba ligado... yo estaba empezando a escribir mis primeros cuentos en aquel momento y entonces hicimos una mesa redonda en la Facultad de Filosofía ligados a una pequeña editora que se llamaba 964 que fue la que editó Palo y hueso, uno de los primeros libros. Hicimos esa mesa para presentar un libro de cuentos de Daniel Moyano, La lombriz, que era el segundo libro de cuentos de él. Hicimos esa mesa redonda en la Facultad de Filosofía y Letras de Buenos Aires y estaba Saer, estaba Moyano, estaba yo, y estaba Roa Bastos que fue siempre muy generoso en su relación con los jóvenes escritores. Todos lo queríamos mucho y lo respetábamos mucho y Roa Bastos fue ahí un poco como para respaldar esa presentación. Entonces ahí se produjo una situación, que creo que es bueno recordarla, primero porque fue la primera discusión o conversación con Saer. Porque en aquel tiempo había una suerte de exposición que Saer encarnaba como pocos, que tendía a valorar una serie de escritores del interior del país, como era el caso de Saer en Santa Fe, de Juan José Hernández en Tucumán, de Moyano que estaba en La Rioja, de Tizón en Jujuy; y entonces había una tensión entre la literatura del interior, como se la llamaba, que es una tensión que siempre ha persistido, y la literatura de Buenos Aires. Pero lo divertido del asunto es que yo, que en realidad había nacido en Adrogué, no había nacido en la ciudad y que en ese momento vivía en La Plata, aparecí como un unitario, yo era como el unitario. Frente a esa suerte de perspectiva federal del interior, que venían a encarnar Moyano y Saer, yo aparecía como el representante de la ciudad de Buenos Aires y del centralismo y de toda la injusticia que suponía la relación entre la literatura que se hacía en Buenos Aires. Yo asumí esa posición, ya que me estaban queriendo hacer aparecer como aquel que debía encarnar esa tradición que todo el mundo criticaba, yo asumí esa situación y entonces se formó una discusión interesantísima. Que por otro lado, Roa Bastos funcionaba como 
el Papa ¿no? era como el Papa Roa, porque Roa desde luego valoraba muchísimo este tipo de literatura porque consideraba que esa literatura estaba conectada con la literatura de América Latina, con cierta razón. Que la literatura que se estaba haciendo en ese momento en ciertas zonas alejadas del núcleo de Buenos Aires estaba más ligada con la literatura de Rulfo, de Guimaraes Rosa, que la literatura que se estaba haciendo en Buenos Aires. Y entonces la discusión tenía al mismo tiempo ahí a Roa como una suerte de árbitro que también estaba comprometido con la situación. Tuvimos esa discusión muy divertida y después desde luego salimos de ahí y nos fuimos a los bares a seguir discutiendo y creo que desde esa discusión seguimos discutiendo durante todos los años de nuestra amistad. Pero lo que quiero señalar es por un lado la escena ¿̨no? una pequeña editora que publica un libro de un escritor joven, como era Moyano en ese momento, y que va a publicar también el libro de Saer, y que crea un debate. Y que es un debate sobre las tradiciones, a esto me refiero. Es un debate sobre las tradiciones porque lo que se estaba discutiendo ahí era si esta tensión entre la literatura que se estaba haciendo en Buenos Aires y la literatura que se hacía en otras periferias, en otras áreas, podía o no entenderse todo como literatura argentina. O si teníamos que hacernos cargo de la tensión que esto estaba generando. Me parece que la obra de Saer está ligada a la discusión sobre esa tradición, ligada a lo que podríamos considerar esta idea de la región y por lo tanto cierta tensión con el regionalismo. La idea de que la región es el lugar desde el cual hay que leer la literatura mundial, es decir, que la literatura siempre debe ser leída desde un espacio específico. Siempre hay que leerla desde un lugar determinado y que ese lugar, que es el lugar de apropiación, que es el lugar a partir del cual uno lee las otras tradiciones, uno no debe identificarse con las otras tradiciones, sino que debe traer esas tradiciones a su propio espacio y que debe leerlas en su propio espacio para resolver los problemas que su propia literatura está generando, y eso es lo que hace Saer cuando lee Pavese, cuando lee Joyce o cuando lee Faulkner. Los lee como si fueran escritores que están pensando y tratando de narrar el mismo tipo de problemas que él se está planteando cuando intenta ver si es posible construir una historia que tenga a Santa Fe y al litoral como nudo. Y por lo tanto eso le permite leer un conjunto de textos, pero es una lectura situada. La idea de que la literatura debe estar localizada y espacializada y que el debate debe ser un debate que se da en un espacio definido, es algo que Saer ha planteado siempre como un modo de entender la noción de tradición. La noción de tradición quiere decir por un lado que las tradiciones deben ser leídas cuando están localizadas y que al localizarse en un lugar definido, cambia el uso que podemos nosotros. Nosotros no leemos a Faulkner igual a como lo leen en Nueva York, o nosotros no leemos a Faulkner como lo lee un escritor norteamericano. Leemos otra cosa ahí, estamos usando esos textos en relación al intento de construir tradiciones propias...

Vive en Santa Fe y después directamente se va a Paris. Entonces esa manera de esquivar Buenos Aires nos podría hacer a nosotros pensar que en realidad lo que Saer está tratando de pensar es un tipo de noción que no incluya el concepto literatura nacional, que no se haga cargo del concepto de literatura nacional. Que trabaje con esta noción de una literatura situada en una región que se conecta con la literatura mundial, sin pasar por la mediación de la literatura nacional y de todo lo que la literatura nacional trae.

Porque la literatura nacional era vista como una sola literatura, como si la literatura nacional fuese de hecho un único modo de entender las circulaciones 
de los textos y los usos de las tradiciones, etc. Me parece que incluso en el gesto, en la historia, en el gesto que podemos leer casi como una alegoría de un escritor que vive en Santa Fe y se forma en Santa Fe y luego va al centro, va a Paris, pero esquiva Buenos Aires, ahí podríamos leer también un signo casi inesperado de lo que podríamos entender como un punto de partida para percibir el sentido que tiene la poética en la narración en Saer.

En ese sentido me interesa mucho insistir sobre la noción de tradición y sobre el lugar de Saer en la tradición, porque me parece que tenemos que regresar a la noción de tradición y enfrentar la noción de canon. La noción de tradición es totalmente antagónica con la noción de canon. La noción de canon tiende a fijar y a cristalizar, en un momento determinado, una lista de autores que se supone que aparecen como aquellos que debemos aceptar como tales. Mientras que la noción de tradición supone un lugar de tensión, las tradiciones que se enfrentan entre sí, las poéticas que se enfrentan entre sí, son lugares mucho más inestables. Las relaciones que se establecen entre los textos y los autores empiezan a jugar en el interior de esos espacios dentro de los cuales la literatura actúa y por lo tanto me parece que debemos regresar a la noción de tradición y debemos descartar la noción de canon, que me parece que está hoy en manos de aquellos que buscan guiarse en la literatura con una lista de nombres que les permitan enfrentar lo que aparece como la confusión que el mercado construye. En la confusión que el mercado construye, la noción de canon vendría como a poner orden.

La noción de tradición me parece una noción mucho más dinámica, por supuesto, mucho más conectada con la lentitud, conectada con la relación con el pasado, como una relación que está siempre actuando. Mientras que lo que nosotros asistimos en la cultura actualmente es a una suerte de urgencia por la novedad, una especie de actualidad excesiva. Y frente a esa idea de la actualidad excesiva, debemos, me parece a mí, volver a pensar en la noción de tradición como una manera de utilizar lo que se acumuló en la cultura, lo que hemos hecho hasta aquí y a partir de lo cual podemos insistir en qué es lo que nosotros pensamos que es posible escribir, ¿no es verdad?

También la noción de tradición no se opone solo a esta noción, que está de moda ahora, de canon, sino que también se opone a esta suerte de velocidad de la novedad e intenta establecer una temporalidad que sea una temporalidad propia de la literatura y de la cultura y que no responda a una velocidad de la circulación que no forma parte de la lógica misma de la cultura y de la literatura. Saer, me parece, tenía muy clara esta cuestión.

El otro elemento importante de la noción de tradición es que la tradición viene a poner el debate sobre la literatura desde el punto de vista del sujeto que está haciendo la literatura. Es decir, la tradición está construida por los propios escritores, son los escritores quienes luchan y enfrentan textos, descartan algunos e incluyen otros y por lo tanto la noción de tradición está muy conectada con la lucha de poéticas, con los espacios dentro de los cuales los escritores actúan y es por lo tanto también un modo interesante de plantear el problema de la historia literaria. La tradición es en cierto sentido antagónica con la historia literaria porque está todo el tiempo revisando la historia literaria y está todo el tiempo revalorando textos que han sido perdidos y se vuelven a recuperar, y se vuelven a reconstruir las tradiciones, y por lo tanto en ese sentido la noción de tradición es una noción que surge de la propia dinámica que los escritores le dan a la discusión sobre lite- 
ratura. Y por otro lado podríamos decir que todo ese tipo de cuestiones que giran alrededor de estas cuestiones que estamos viendo, están también en las novelas. No se trata solamente de posiciones teóricas. Nosotros podríamos reconstruir una línea, una tradición, un modo de pensar la literatura argentina a partir de ciertas novelas. Podríamos leer El juguete rabioso de Roberto Arlt y ver un modo de ficcionalizar el acceso a la cultura, el tipo de dificultades que aparecen ligadas al modo en que es posible construir una cultura alternativa, una cultura de la pobreza, una cultura hecha desde el que viene del margen, una cultura que en cierto sentido está siendo construida con todos los elementos de los márgenes que Arlt trabaja. O podríamos tomar como otro ejemplo The Buenos Aires affair de Manuel Puig y podríamos también ver esa novela como un debate sobre la tradición. Esa artista que se enfrenta con el crítico, que tiene una relación absolutamente paranoica entre la artista y el crítico que es un poco el nudo de esa novela policial de Puig y la idea del artista que hace su obra recogiendo desechos en la basura, lo que deja el mar. Esa artista de la novela de Puig que está trabajando siempre con aquello que sobra, con aquello que ha quedado como fuera de la circulación, podríamos nosotros ver ahí, casi de modo transparente, funcionar a la poética de Puig que en ese momento está haciendo lo mismo y está trabajando con los restos de la cultura. Está trabajando con aquello que la tradición de la biblioteca ha dejado de lado y que son ciertas tradiciones que vienen del cine.

Quiero decir con esto, entonces, que en la literatura también es posible leer las marcas de esta discusión sobre los espacios, la circulación, las tradiciones. Y desde luego la obra de Saer es un laboratorio de este tipo de cuestiones, ¿̇no es cierto? Está todo el tiempo circulando en las novelas de Saer este tipo de problemas. Es en ese sentido también que me refiero al lugar de Saer, me refiero también a ¿cuál es el lugar de Saer en la tradición? ¿qué tipo de tradición es la que él construye? Me parece que ahí, tal vez podríamos pensar a Saer junto con otros escritores que son, no digo antagónicos, pero que tienen poéticas muy diferenciadas, en relación con Saer, y que podríamos pensarlas como poéticas post-Borges, para hablar rápido. Pienso básicamente que la cuestión de los problemas de tradición que Saer plantea, es la tensión entre lo que podríamos llamar literatura y los massmedia. Es decir, el modo en que la presencia de los massmedia ha alterado la noción de tradición y ha construido un espacio de circulación de la cultura a una velocidad que pone en crisis todas las formas de conectarse con la circulación de las tradiciones. Y entonces uno podría ver la obra de Saer, la obra de Puig y la obra de Rodolfo Walsh, como tres respuestas internas a esta tensión entre literatura y cultura de masas. Y si tomamos este tema en relación a estas obras, en principio podríamos ver a Saer respondiendo con una posición de ruptura tajante respecto a todo lo que podríamos entender como el mundo de la cultura de masas. Digamos que la poética de Saer es una poética que agudiza la escisión, que pone frente a la trivialización, como diría él, de los lenguajes que la cultura de masas postula. Saer ve a la literatura como un modo de resistencia a ese tipo de circulación estereotipada del lenguaje. Y entonces establece un tipo de tensión continua entre lo que sería el trabajo de la literatura y ese otro trabajo de esta suerte de circulación estereotipada de los media...

¿En qué lugar Saer construye esa poética? Me parece que la construye en el punto en donde él ve el lugar crítico de la cuestión: el lenguaje. Lo que Saer está diciendo es que el lugar donde se afecta de una manera más nítida esta presencia de la cultura de masas en la cultura contemporánea, es en el modo en que el len- 
guaje es tergiversado, el lenguaje es obligado a decir, de cierta manera, cierto tipo de contenidos. No solo los contenidos sino esta idea de cómo debe circular una suerte de lenguaje estereotipado.

Yo pienso siempre en lo que Pasolini decía hablando de la situación de la lengua italiana. Que hay una lengua media, decía él. Que se ha construido una suerte de lengua media que es una lengua que no es de nadie y que funciona como un espacio al interior del cual se supone que circula lo que podríamos llamar «lo que se comprende». Y todo lo que no pasa por allí queda ajeno a esta lógica de la comprensión.

Mientras que Saer pone en primer plano el problema de ¿qué quiere decir entender? ¿qué quiere decir entender literatura? Es como preguntarse qué quiere decir entender una pasión, no es del orden del entender, es del orden de la transmisión de un determinado tipo de sentimiento o de pasión y no debe entrar a funcionar en los términos en que funcionaría una noción de la comprensión, que está más conectada a otro tipo de lenguajes sociales. En ese sentido me parece que el punto donde esto encuentra su nudo es en la tensión entre narración e información. Me parece que Saer tiene una muy clara idea de que la narración es antagónica con la información. Que la narración es una experiencia que el sujeto hace y que la información es algo que aleja al sujeto de la experiencia, sencillamente el sujeto recibe un conjunto de informaciones determinadas pero esas informaciones están completamente aparte de su propia experiencia. Mientras que la narración sería un modo de transmitir a partir de la experiencia misma. Entonces la tensión narración e información podría ser un punto a partir del cual podríamos nosotros discutir este tipo de posición en el caso de Saer. Entonces, en el momento en que la lengua se ha vuelto opaca y homogénea, el trabajo detallado, microscópico, de la literatura, sería un modo de intervenir incluso políticamente en el mundo social. Sería un modo de entrar con los medios específicos a discutir algo que está ligado a lo que podríamos llamar «La crisis del lenguaje». Es una cuestión a lo que los escritores se han referido desde siempre, diría yo: los usos del lenguaje, la construcción de la creencia, los modos en que se construye determinado tipo de verdad a partir de cierto tipo de manipulación del lenguaje. A mí siempre me ha parecido que no es nada casual que el mayor crítico político actual sea un lingüista. El hecho de que Chomsky aparezca hoy como uno de los críticos más claros respecto a la situación política, no debe descartar el hecho de que es un lingüista, es decir, alguien que tiene mucha experiencia en el análisis del lenguaje. Y que a partir de su experiencia en el análisis del lenguaje es capaz de empezar a descifrar el funcionamiento del poder.

Creo que a Saer podríamos ponerlo en esa cuestión. Es el lenguaje el lugar a partir del cual lo social empieza a actuar y por lo tanto la literatura tiene una intervención en esta cuestión. Y me parece que el punto donde Saer ve el lugar desde el cual es posible ver el horizonte de una utilización distinta del lenguaje es desde luego, la poesía. La poesía sería ese lugar donde el lenguaje es usado de un modo que es totalmente ajeno a los usos instrumentales que van desde el discurso político, los usos instrumentales que tienen que ver con el discurso político, con el discurso del Estado, con el discurso de los medios. Frente a eso aparecería la experiencia de la poesía como una experiencia donde el lenguaje preserva esa posibilidad de hablar del sentido, esa posibilidad de aludir al sentido, esa posibilidad de aludir a una experiencia que no es una experiencia que pase por esa suerte de estabilidad ¿no? Como saben ustedes, hay que decir las cosas de modo que todo el mundo las 
entienda pero eso quiere decir decirlas de un modo determinado. Empieza todo un sistema que suele invadir el debate cultural. Como si hubiera una suerte de superioridad por el cual habría que bajar el nivel, como si en cierto sentido siempre tuviéramos que pensar que estamos hablando en una situación escolar. Tenemos que poner el lenguaje a la altura, y mientras tanto la experiencia es al revés. Es en el lenguaje hablado, es en la experiencia de los relatos que circulan en la ciudad, es en las conversaciones donde está la materia prima a partir de la cual la poesía y la literatura construyen sus usos del lenguaje. Esta tensión entre la poesía por un lado y los media por otro es un punto de partida para entrar en esta cuestión del lugar de Saer. Y les voy a leer una cita de 1969. En el texto La literatura y los nuevos lenguajes, Saer decía esto: «Se puede decir que al menos desde 1950 los massmedia instalaron en el lugar de la escritura, el reino del estereotipo del arte de segunda mano de la tautología oficial, de la fraseología hueca que repite con una servilidad calculada las simplificaciones de los verdugos y de los mercaderes». E inmediatamente agrega, definiendo el otro aspecto: «De ahí que el ejemplo privilegiado de la literatura sea la poesía, y en especial la poesía lírica, y que entre la poesía lírica y los massmedia las relaciones sean totalmente inexistentes».

Es decir que si queremos empezar a entrar en «el lugar de Saer» y si empezamos a pensar en qué tradición quiere instalar sus textos, tenemos que partir de esta tensión, de aquello de lo que se quiere alejar, aquello frente a lo cual se está construyendo la alternativa.

\footnotetext{
Notas

${ }^{1}$ Transcripción realizada por Pamela Silva y Josefina Ganuza

${ }^{2}$ El contenido de las conferencias y paneles del III Argentino de Literatura será editado en un ebook, subido próximamente al sitio web del Centro de Estudios Comparados. http://www.fhuc.unl.edu.ar/pages/investigacion/ centros-institutos-y-laboratorios/centro-de-estudios-comparados-cec.php ${ }^{3}$ Diálogo Piglia-Saer. Por un relato futuro (1990) Santa Fe: Centro de Publicaciones UNL. Republicado en 1995.
} 